\title{
The DIANA Underground Accelerator Facility Project at DUSEL Laboratory
}

\author{
Alberto Lemut ${ }^{* i a}$ Manoel Couder, ${ }^{b}$ Daniel Winklehner, ${ }^{a, c}$ Arthur E. Champagne, ${ }^{d}$ \\ Philippe Collon, ${ }^{b}$ Michael Famiano, ${ }^{e}$ Fredrick Gray, ${ }^{f}$ Uwe Greife, ${ }^{g}$ Adrian \\ Hodgkinson, ${ }^{a}$ Christian Iliadis, ${ }^{d}$ Daniela Leitner ${ }^{a, c}$ Mattheaus Leitner, ${ }^{a, c}$ Joseph S. \\ Saba, ${ }^{a}$ Edward Stech, ${ }^{b}$ Paul A. Vetter, ${ }^{a}$ William L. Waldron, ${ }^{a}$ and Michael Wiescher ${ }^{b}$ \\ ${ }^{a}$ Lawrence Berkeley National Laboratory, 1 Cyclotron Rd, Berkeley 94720 CA, USA \\ ${ }^{b}$ University of Notre Dame, Notre Dame, IN, USA \\ ${ }^{c}$ Michigan State University, East Lansing, MI, USA \\ ${ }^{d}$ University of North Carolina at Chapell Hill, Chapell Hill, NC, USA \\ ${ }^{e}$ West Michigan University, Kalmazoo, MI, USA \\ ${ }^{f}$ Regis University, Denver, CO, USA \\ ${ }^{g}$ Colorado School of Mines, Golden, CO, USA \\ E-mail: ALemut@lbl.gov
}

The DIANA project (Dakota Ion Accelerators for Nuclear Astrophysics) is a collaboration between the University of Notre Dame, Colorado School of Mines, Regis University, University of North Carolina, Western Michigan University, and Lawrence Berkeley National Laboratory, to build a next generation nuclear astrophysics accelerator facility deep underground. The DIANA accelerator facility is being designed to achieve large laboratory reaction rates by delivering high ion beam currents (up to $100 \mathrm{~mA}$ ) to a high density (up to $10^{18}$ atoms $/ \mathrm{cm}^{2}$ ), super-sonic jet-gas target. The accelerator developments of the DIANA facility are presented here.

11th Symposium on Nuclei in the Cosmos, NIC XI

July 19-23, 2010

Heidelberg, Germany

\footnotetext{
*Speaker.

${ }^{\dagger}$ This work was supported by the National Science Foundation NSF-09-500 grant (DUSEL S4), Proposal ID 091728
} 


\section{Introduction}

Current stellar model simulations are at a level of precision such that nuclear-reaction rates represent a major source of uncertainty for theoretical predictions and for the analysis of observational signatures [1]. To address several open questions in cosmology, astrophysics, and non-StandardModel neutrino physics, new high precision measurements of direct-capture nuclear fusion cross sections will be essential [1]. Experimental studies of nuclear reaction of astrophysical interest are hampered by the exponential drop of the cross-section [2]. The extremely low values of $\sigma(E)$ within the Gamow peak prevents its measurement in a laboratory at the earth surface. The signal to noise ratio would be too small, even with the highest beam intensities presently available from industrial accelerators, because of the cosmic ray interactions with the detectors and surrounding materials.

An excellent solution is to install an accelerator facility deep underground where the cosmic rays background into detectors is reduced by several order of magnitude [3], allowing the measurements to be pushed to far lower energies than presently possible. This has been clearly demonstrated at the Laboratory for Underground Nuclear Astrophysics (LUNA) [4] by the successful studies of critical reactions in the pp-chains [5] and first reaction studies in the CNO cycles [6]. However many critical reactions still need high precision measurements [1], and a next generation facility, capable of very high beam currents over a wide energy range and state of the art target and detection technology, is highly desirable.

The DIANA accelerator facility is being designed to achieve large laboratory reaction rates by delivering high ion beam currents (up to $100 \mathrm{~mA}$ ) to a high density (up to $10^{18}$ atoms $/ \mathrm{cm}^{2}$ ), supersonic jet-gas target as well as to a solid target. DIANA will consist of two accelerators, $50-400 \mathrm{kV}$ and 0.4-3 MV, that will cover a wide range of ion beam intensities, with sufficient energy overlap to consistently connect the results to measurements above-ground. Currently DIANA is proposed to be a part of the of the Integrated Suite of Experiments (ISE) of the US proposal DUSEL (Deep Underground Science and Engineering Laboratory) to establish a inter-disciplinary underground laboratory in the former gold mine of Homestake in South Dakota, USA. In particular, three fundamental scientific issues in stellar nucleosynthesis will be addressed by DIANA: (i) solar neutrino sources and the metallicity of the sun; (ii) carbon-based nucleosynthesis; and (iii) neutron sources for the production of trans-Fe elements in stars. The design of the DIANA accelerator facility is presented here.

\section{DIANA accelerator developments}

The DIANA accelerator facility will consist of two accelerators: $50-400 \mathrm{kV}$ and $0.4-3 \mathrm{MV}$ (Fig. 1). Independent solid and jet target stations are under design for both accelerator for conducting two experimental campaigns simultaneously or preparing the next experimental campaign (Fig. 1). This feature will greatly enhance the ability to carry out the planned science program timely and efficiently, since the experimental setups are difficult and time consuming. Moreover the high energy accelerator will have the capability to provide beam to the low energy target stations (Fig. 1). This will allow a particular reaction to be measured with both accelerators in complementary energy ranges with identical target and detector setups, providing consistent high-precision data 


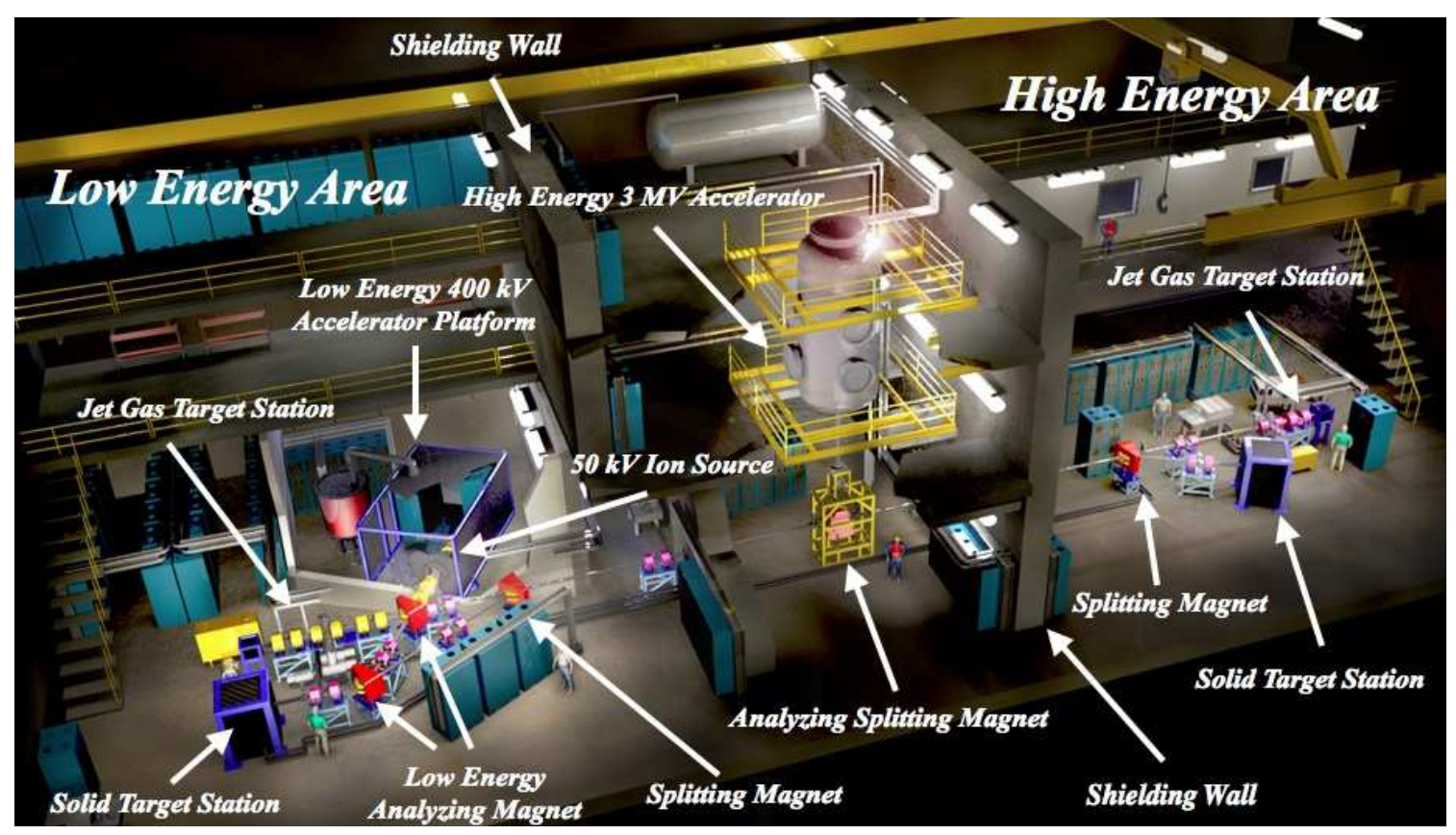

Figure 1: View of the DIANA accelerator facility.

over a wide energy range. Very intense ion-beams (10 to $100 \mathrm{~mA}$ for the $400 \mathrm{kV}$, up to $1 \mathrm{~mA}$ for the $3 \mathrm{MV}$ ), continuously over long period of time, are required in order to address the low count rates close to the Gamow window energies. In addition tight focus $(<1 \mathrm{~cm})$ at the jet-gas target stream, and narrow beam energy resolution (about $2.5 \times 10^{-4}$ ) are required.

The ion source currently considered for the low energy machine is a microwave one based on the Chalk River development [7]. Since these ion sources require no filament, they are very low maintenance and can run uninterruptedly for extended period of time. The low energy accelerator consists of a $400 \mathrm{kV}$ open air platform (Fig. 1). It is being designed to extract all beams at $50 \mathrm{kV}$ and post-accelerate them up to $400 \mathrm{kV}$. This approach allows for the maximum optimizations of the ion source independent of the required final beam energy, allowing for state of the art ion source design. The extraction from an aperture of $9 \mathrm{~mm}$ diameter of $100 \mathrm{~mA}, 50 \mathrm{keV}^{4} \mathrm{He}^{+}$ and ${ }^{1} \mathrm{H}^{+}$beams has been reported [8]. The transport of such high currents at low ion energies is made challenging because of the rapid beam expansion over short distances due to the beam self space charge repulsion. To overcome this problem the low energy accelerator beam transport system incorporates several $2 \mathrm{~T}$ solenoid lenses to maintain the beam radius to an acceptable value in particular while passing through the analyzing magnet gap, and at the jet gas target stream where a tight focus is required. The analyzing magnet has been designed to separate the beam of interest from any impurity present in the beam. Results of a beam optics calculation, are shown in Fig. 2, showing the feasibility of such a low energy high current accelerator.

The high energy accelerator possibilities under consideration are commercially available pelletrons or dynamitrons. The ion source currently proposed for the high energy machine is an Electron Cyclotron Resonance (ECR) ion source, capable of producing high intensity beams, up to $5 \mathrm{~mA}$ for single charged ions, and tenths of $\mu A$ of medium charge state heavier ions. In order to get relatively 

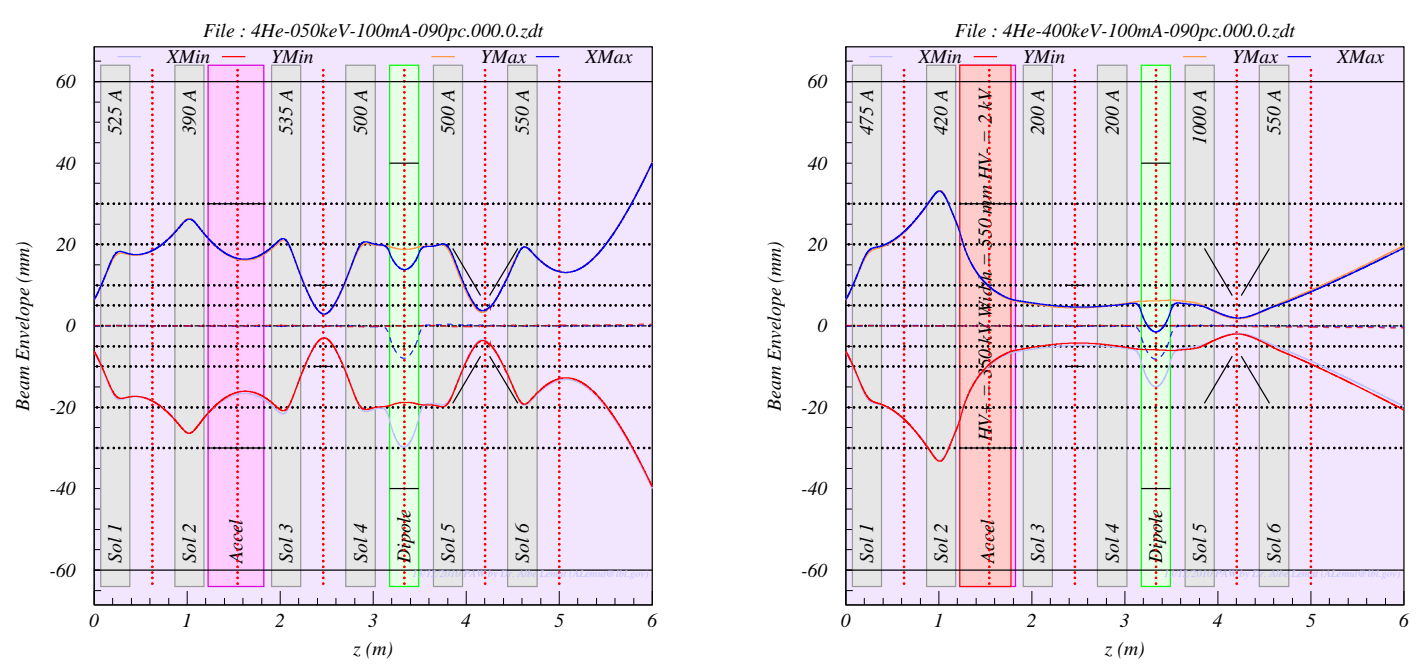

Figure 2: Left: beam envelopes for a $50 \mathrm{keV}, 100 \mathrm{~mA}{ }^{4} \mathrm{He}^{+}$beam. Right: beam envelopes for a $50 \mathrm{keV}$, $100 \mathrm{~mA}^{4} \mathrm{He}^{+}$initial beam post-accelerated to $400 \mathrm{keV}$. Space charge effects have been taken into account in the calculation ( $90 \%$ neutralisation was assumed along the beam line, $0 \%$ inside the accelerator tube when it is powered). It should be noted that in both cases the beam diameter at target, $z=4.2 \mathrm{~m}$, is $<1 \mathrm{~cm}$.

high ion currents at the lowest accelerating voltages, with a beam radius of a few millimeters, the accelerator tube of the high energy machine will need to be custom designed. In particular to reduce the beam expansion in the accelerator tube, the Shorting Rod (SR) technique has been widely adopted since. In this technique, for every voltage applied to the column, an optimum number of active ring is set, while the remaining rings are tied to ground by using a movable SR. In a standard accelerator column design the voltage is graded uniformly along the accelerator column. In most designs, the gradient along the column is set to the maximum value allowed by sparking. As a consequence a strong focusing effect is built at the entrance of the accelerator tube, where the particles start to be accelerated, causing the beam to scrape the tube after a short path, reducing the transported beam intensity and generating secondary electrons. A widely adopted solution is to install an Einzel Lens (EL) to compensate for this strong focusing effect. Coupling the EL with the SR technique, good beam intensity are obtained at high accelerating voltages. However at low energies (critical for nuclear astrophysics studies) the beam expands rapidly because of the space charge, causing the beam to scrape the tube, and reducing the current transported by the tube. Furthermore the final beam diameter will be too high to be efficiently transported in the beam lines by the focusing elements. To reduce the strong focusing effect, an alternative approach is to use the Variable Voltage Gradient (VVG) technique, where the voltage is graded smoothly along the column. If a resistor chain is used to set the voltage, this can be easily achieved by tuning each resistor to an appropriate value. At low energies the beam will be accelerated by a small electric field, which increase along the column as the beam energy increase. The VVG technique coupled with the SR, will reduce the particle scraping along the column, transporting all the beam up to the tube exit. However the final diameter will still be inconsistent at different energies if the intensities are not scaled with final beam energy. To reduce this effect the column will be equipped with an 

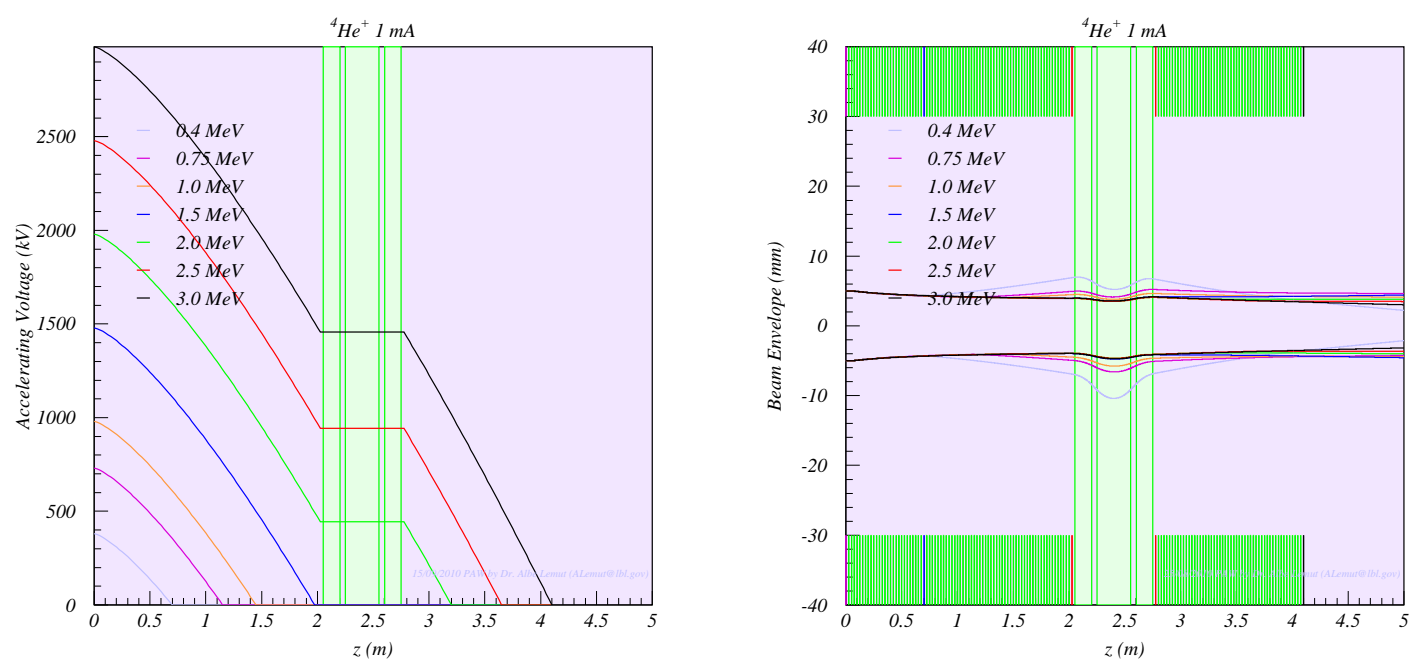

Figure 3: Left: VVG voltage distribution along the column, except for the EQT region, where it is constant. The position of the Shorting Rod, for each of the selected final energies, is where the voltage becomes zero. Right: beam envelopes for a $1 \mathrm{ma}^{4} \mathrm{He}^{+}$for selected final beam energies. Space charge effect are taken into account: the beam has been assumed $0 \%$ neutralised inside the column and $90 \%$ outside. Thanks to the embedded EQT the final beam diameter is less than $1 \mathrm{~cm}$. To further reduce the beam diameter before entering the analyzing magnet, an other focusing element will be added.

embedded Electrostatic Quadrupole Triplet (EQT), installed in the middle of the column, providing additional focusing. The result of ion beam optics calculation for a column design combining VVG, SR, and EQT techniques is shown on the right side of Fig. 3 for several final beam energies and a constant ion beam of $1 \mathrm{~mA}$. The final beam diameter is few millimeters and can be easily transported through the high energy beam lines for all the energies.

\section{References}

[1] E. G. Adelberger et Al., arXiv:1004.2318v2 (2010).

[2] C. Rolfs, W. S. Rodney, Cauldrons in the Cosmos, University of Chicago Press (1988).

[3] D. M. Mei and A. Hime, Phys Rev. D 73 (2006) 053004.

[4] H. Costantini et Al, Rep. Prog. Phys. 72 (2009) 086301.

[5] M. Junker et Al., Phys. Rev. C 57 (1998) 2700.

[6] A. Lemut et Al, Phys. Lett. B 634 (2006) 483.

[7] T. Taylor and J. S. C. Willis, Nucl. Instr. Meth A 309 (1991) 37.

[8] R. Gobin et. Al., Proceeding of the ECRIS 2008 conference, Chicago, USA (2008). 